\title{
A COMPACT TOPOLOGY FOR A LATTICE ${ }^{1}$
}

\author{
ARNOLD J. INSEL
}

Introduction. In this paper we shall study a compact intrinsic topology for a lattice and obtain a few relationships between this topology and certain well-known intrinsic topologies for lattices. We obtain as a result the fact that for a large class of lattices, compactness of the order topology implies that our compact topology and the order topology coincide.

Let $L$ be a lattice and $\left\{x_{a}\right\}$, a net in $L$. We define the limit inferior, $L_{*}\left\{x_{a}\right\}=\bigvee_{a} \Lambda_{b \geqq a} x_{b}$, and the limit superior, $L^{*}\left\{x_{a}\right\}=\Lambda_{a} \bigvee_{b \geqq a} x_{b}$. Then, provided they exist, $L_{*}\left\{x_{a}\right\} \leqq L^{*}\left\{x_{a}\right\}$. If $L_{*}\left\{x_{a}\right\}=L^{*}\left\{x_{a}\right\}=x$, we say that the net $\left\{x_{a}\right\}$ order converges to $x$. Let $C$ be a subset of $L$. $C$ is said to be order closed iff no net in $C$ order converges to a point outside of $C$. The collection of order closed sets comprises the closed sets for a topology for $L$. We call this topology the order topology for $L$ and designate it by $O(L)$.

The collection of sets of the form $\{x: x \leqq c\}$ and $\{x: x \geqq c\}$ for $c \in L$ forms the sub-base of the closed sets of a weaker topology called the interval topology and designated by $I(L)$. It is known that for any lattice $L, L$ is complete iff $L$ with the interval topology is compact. (See G. Birkhoff's Lattice theory.)

The complete topology. Definition 1 . Let $L$ be a lattice and $\mathfrak{e}$, the collection of all complete subsets of $L$. Then $\mathbb{C}$ is a subbase of the closed sets for a topology which we shall denote by $K(L)$. The topology $K(L)$ will be called the complete topology for $L$.

Lemma 2. Let $L$ be a lattice and $\Re$, a nest of nonempty complete subsets of L. Then $\cap \Re \neq \varnothing$.

Proof. Let $S$ denote the set, $\{\bigvee N: N \in \mathfrak{N}\}$. For the following discussion we consider a fixed set $N_{0}$ in $\Re$. We divide the proof up into several remarks.

Remark (a). Define $S_{0}=\left\{s: s \in S\right.$ and $\left.s \leqq \bigvee N_{0}\right\}$. Then, $S_{0} \subset N_{0}$.

Proof. Consider any element $s \in S_{0}$. If $s=\bigvee N_{0}$ then $s \in N_{0}$, since $N_{0}$ is complete. Suppose $s<\mathrm{V} N_{0}$. Then associated with $s$ is a member $N_{s}$ of $\Re$ such that $s=\mathrm{V} N_{s}$. Then since $\Re$ is a nest, either $N_{0} \subset N_{s}$ or $N_{8} \subset N_{0}$. If $N_{0} \subset N_{8}$ then clearly, $\mathrm{V} N_{0} \leqq \mathrm{~V} N_{8}=s$, contrary to hypothesis. Hence $N_{s} \subset N_{0}$, and since $N_{0}$ is complete, $V_{s}=s \in N_{0}$.

Received by the editors April 2, 1962.

1 The author wishes to express his appreciation to Professor T. O. Moore under whose direction this research was done. 
REMARK (b). $S$ is linearly ordered.

Proof. Let $s$ and $t$ be elements of $S$. Associated with $s$ and $t$ are sets $N_{s}$ and $N_{t}$ in $\mathscr{H}$ such that $s=\mathrm{V} N_{s}$ and $t=\mathrm{V} N_{t}$. Either $N_{s} \subset N_{t}$ or $N_{t} \subset N_{s}$. In the former case $s \leqq t$, in the latter, $t \leqq s$.

REMARK (c). Both $S$ and $S_{0}$ possess infimums, and $\Lambda S=\wedge S_{0}$.

Proof. By Remark (a), $S_{0}$ is contained in a complete subset of $L$ and hence, $S_{0}$ possesses an infimum, $\wedge S_{0}$. Consider any $x \in S$. By Remark (b), we may note that either $x \leqq \mathrm{~V} N_{0}$ or $x>\mathrm{V} N_{0}$. If $x>\mathrm{V} N_{0}$ then since $\mathrm{V} N_{0} \in S_{0}$, we have that $\Lambda S_{0} \leqq \mathrm{~V} N_{0}<x$. Clearly if $x \leqq \bigvee N_{0}$, $x \in S_{0}$ and hence, $\Lambda S_{0} \leqq x$. We conclude that $\Lambda S_{0}$ is a lower bound for $S$. Now suppose that $y$ is any lower bound for $S$. Then since $S_{0} \subset S$, $y$ is a lower bound for $S_{0}$ or, $y \leqq \Lambda S_{0}$. Hence $\Lambda S_{0}$ is the greatest lower bound for $S$ or, $\wedge S_{0}=\wedge S$.

We now continue with our proof. $N_{0}$ is a complete subset of $L$. By Remark (a), $S_{0} \subset N_{0}$ and hence, $\Lambda S_{0} \in N_{0}$. But by Remark (c), $\Lambda S_{0}=\Lambda S$ and hence, $\Lambda S \in N_{0} . N_{0}$ was chosen as an arbitrary member of $\mathscr{N}$ and it was found that $\Lambda S \in N_{0}$. Hence $\Lambda S \in \cap \Re$ or, $\cap \Re \neq \varnothing$.

THEOREM 3. Let a be a collection of complete subsets of a lattice $L$ such that $a$ has the finite intersection property. Then, $\cap \propto \neq \varnothing$.

Proof. We use transfinite induction on the cardinality of $a$. Clearly the theorem holds if $Q$ is finite. Suppose that the theorem holds if the cardinality of $a$ is less than some fixed cardinal number $\alpha$. Let $\Gamma$ be the set of all ordinal numbers less than the first ordinal number of cardinality $\alpha$. Assume the cardinality of $\Gamma$ is the same as that of $a$. Hence we can index $a$ with $\Gamma$. Then for each ordinal $n \in \Gamma$ we consider the set $A_{n}$ in $a$ to which $n$ corresponds. Define the set $C_{n}$ $=\bigcap\left\{A_{i}: i \in \Gamma\right.$ and $\left.i \leqq n\right\}$. Then $C_{n}$ is not empty because it is the intersection of a collection of complete sets with the finite intersection property, and this collection has cardinality less than $\alpha$. Thus the collection $\left\{C_{n}: n \in \Gamma\right\}$ forms a nest of nonempty complete subsets of $L$. Hence by Lemma $2, \cap\left\{C_{n}: n \in \Gamma\right\}=\cap \propto \neq \varnothing$.

TheOREM 4. Let $L$ be a lattice with the complete topology, $K(L)$. Then $L$ is compact.

Proof. The lattice $L$ with the topology $K(L)$ satisfies the following property: There exists a subbase of the closed sets for $K(L)$ such that every subcollection of this subbase with the finite intersection property has a nonempty intersection. But this condition is necessary and sufficient for $L$ with the topology $K(L)$ to be compact. (For a proof see O. Frink's Topology in lattices.)

TheOREm 5. Let $L$ be a lattice. Then $K(L) \subset O(L)$, and $I(L) \subset K(L)$ iff $L$ is complete. 
Proof. Clearly every complete subset of $L$ is order closed and hence, $K(L) \subset O(L)$. If $I(L) \subset K(L)$, then since $K(L)$ is compact, $I(L)$ is compact and hence, $L$ is complete. If on the other hand, $L$ is complete, each closed ray of the form $\{x: x \leqq c\}$ or $\{x: x \geqq c\}$ is complete and hence, $I(L) \subset K(L)$.

Let $L$ be a complete lattice and $A$, a nonempty subset of $L$. Then by $A^{q}$ we shall mean the smallest complete subset of $L$ containing $A$. If $\left\{x_{a}\right\}$ is a net in $L$, by $\left\{x_{a}\right\}^{a}$ we shall mean the smallest complete subset of $L$ containing the range of the net. The following is a characterization of topological convergence in a complete lattice with respect to the complete topology, $K(L)$.

THEOREM 6. Let $L$ be a complete lattice and $\left\{x_{a}\right\}$, a net in $L$. Then $\left\{x_{a}\right\}$ topologically converges to a point $x$ in $L$ with respect to the complete topology, $K(L)$, iff for each subnet $\left\{y_{c}\right\}$ of $\left\{x_{a}\right\}, x \in\left\{y_{c}\right\}^{q}$.

Proof. Suppose $\left\{x_{a}\right\}$ converges to a point $x$ in $L$ with respect to the complete topology. Let $\left\{y_{c}\right\}$ be an arbitrary subnet of $\left\{x_{a}\right\}$. Then $\left\{y_{c}\right\}$ converges to $x$. The set $\left\{y_{c}\right\}^{a}$ is closed and contains the net $\left\{y_{c}\right\}$. Hence, $x \in\left\{y_{c}\right\}^{q}$.

Now suppose that $\left\{x_{a}\right\}$ does not converge to $x$. Then there exists a complete subset $C$ of $L$ such that $\left\{x_{a}\right\}$ is frequently in $C$ and $x \notin C$. Hence there exists a subnet $\left\{z_{b}\right\}$ of $\left\{x_{a}\right\}$ contained in $C$. Therefore $\left\{z_{b}\right\}^{a}$ is contained in $C$. Hence $x \in\left\{z_{b}\right\}^{q}$.

Corollary 7. Let $\left\{x_{a}\right\}$ be a net in a complete lattice $L$. Then the set of all elements in $L$ to which $\left\{x_{a}\right\}$ converges with respect to $K(L)$ is a complete subset of $L$.

CoROllaRy 8. Let $L$ be a complete lattice and $\left\{x_{a}\right\}$, a net in $L$. Then if $\left\{x_{a}\right\}$ topologically converges to a point $x$ in $L$ with respect to the complete topology, $K(L), L_{*}\left\{x_{a}\right\} \leqq x \leqq L^{*}\left\{x_{a}\right\}$.

Proof. We first note that for $A$, a nonempty subset of $L, \bigvee A$ $=\bigvee A^{q}$, and $\Lambda A=\Lambda A^{q}$.

For any element $a$ of the directed set of $\left\{x_{a}\right\}, x \in\left\{x_{b}: b \geqq a\right\}$. Hence, for any $a, \Lambda_{b \geqq a} x_{b} \leqq x$. Therefore, $\vee_{a} \Lambda_{b \geqq a} x_{b}=L_{*}\left\{x_{a}\right\} \leqq x$. Dually, $x \leqq L^{*}\left\{x_{a}\right\}$.

TheOREM 9. Let $L$ be a complete lattice and $T(L)$, any compact topology for $L$. Then if every complete subset of $L$ is closed with respect to the topology $T(L), T(L)$ is contained in the order topology, $O(L)$.

Proof. If every complete subset of $L$ is closed with respect to the topology $T(L)$, we have that $K(L) \subset T(L)$. Hence, topological convergence with respect to $T(L)$ implies topological convergence with respect to $K(L)$. Therefore by the previous corollary, if $\left\{x_{a}\right\}$ is a net in $L$ and $\left\{x_{a}\right\}$ converges to a point $x$ with respect to the topology 
$T(L), L_{*}\left\{x_{a}\right\} \leqq x \leqq L^{*}\left\{x_{a}\right\}$.

Now let $C$ be any subset of $L$ such that $C$ is not closed with respect to $O(L)$. Then there exists a net, $\left\{x_{a}\right\}$ in $C$ which order converges to a point $x$, not in $C$. But since $L$ is compact with respect to $T(L)$, there exists a subnet $\left\{y_{b}\right\}$ of $\left\{x_{a}\right\}$ and an element $y$ in $L$ such that $\left\{y_{b}\right\}$ topologically converges to $y$ with respect to $T(L)$. Hence, $L_{*}\left\{x_{a}\right\} \leqq L_{*}\left\{y_{b}\right\} \leqq y \leqq L^{*}\left\{y_{b}\right\} \leqq L^{*}\left\{x_{a}\right\}$. But $L_{*}\left\{x_{a}\right\}=L^{*}\left\{x_{a}\right\}=x$. Hence, $x=y$. Therefore, $y \notin C$. Thus there exists a net in $C$ which converges to a point, $y$, outside of $C$. Therefore $C$ is not closed in the $T(L)$ topology. Therefore, $T(L) \subset O(L)$.

THEOREM 10. Let $L$ be any lattice which satisfies the following condition: If $\left\{x_{a}\right\}$ is a net in $L$ which topologically converges to a point $x$ with respect to the order topology, then there exists a subnet of $\left\{x_{a}\right\}$ which order converges to $x$.

Then if $L$ is compact with respect to its order topology, $O(L)$, it follows that $O(L)=K(L)$.

Proof. Suppose that $L$ satisfies the above condition and suppose further that $L$ is compact with respect to $O(L)$. Then since $I(L)$ $C O(L), I(L)$ is compact and therefore, $L$ is complete.

Let $C$ be a subset of $L$ such that $C$ is not closed with respect to $K(L)$. Then there exists a net $\left\{x_{a}\right\}$ in $C$ and a point $x \notin C$ such that $\left\{x_{a}\right\}$ converges to $x$ with respect to the topology $K(L)$. Since $L$ is compact with respect to $O(L)$, there exists a subnet $\left\{y_{b}\right\}$ of $\left\{x_{a}\right\}$ and a point $y$ in $L$ such that $\left\{y_{b}\right\}$ topologically converges to $y$ with respect to the order topology. By hypothesis, there exists a subnet $\left\{z_{c}\right\}$ of $\left\{y_{b}\right\}$, and hence of $\left\{x_{a}\right\}$, which order converges to $y$. The net $\left\{z_{c}\right\}$ topologically converges to $x$ with respect to $K(L)$. Hence by Corollary $8, L_{*}\left\{z_{c}\right\} \leqq x \leqq L^{*}\left\{z_{c}\right\}$. However, $L_{*}\left\{z_{c}\right\}=L^{*}\left\{z_{c}\right\}=y$. Therefore, $x=y$. Hence, $y \notin C$. We conclude that $C$ is not closed in the order topology for $L$. Hence, $O(L) \subset K(L)$. But $K(L) \subset O(L)$. Hence the theorem follows.

For any lattice $L$ it can be shown that if $L$ is Hausdorff with respect to the complete topology for $L$ then $L$ is a complete lattice. The following questions remain unanswered:

1. Does Theorem $\mathbf{1 0}$ hold for arbitrary lattices?

2. For any lattice $L$, is a necessary and sufficient condition for $O(L)$ to be compact that $K(L)$ be Hausdorff?

\section{BIBLIOGRAPHY}

1. Garrett Birkhoff, Lattice theory, Amer. Math. Soc. Colloq. Publ. Vol. 25, Amer. Math. Soc., Providence, R. I., 1948.

2. O. Frink, Topology in lattices, Trans. Amer. Math. Soc. 51 (1942), 569-582.

3. B. C. Rennie, Theory of lattices, Foester and Jagg, Cambridge, 1951.

UnIVERSITY OF FLoRIDA 